\title{
Photomotor Response Data Analysis Approach to Assess Chemical Neurotoxicity with the Zebrafish Embryo
}

\author{
Julia Ortmann, Rolf Altenburger, Stefan Scholz and Till Luckenbach \\ Department Bioanalytical Ecotoxicology, Helmholtz Centre for Environmental Research (UFZ), Leipzig, Germany
}

\begin{abstract}
The photomotor response (PMR) of zebrafish embryos, a light pulse-triggered undirected movement, is known to be altered by neuroactive chemicals. Here, we developed an approach for data analysis of the distribution of PMR movement activities along the time axis; differences between treatment and respective controls are expressed by an aggregated value integrating the time-resolved density of the movement parameter as a measure for a chemically elicited PMR effect. Logistic concentration-PMR effect relationships were modeled for neuroactive test compounds with different modes of action (acetylcholinesterase inhibition, activation and inhibition of voltage-gated sodium channels); $50 \%$ effect concentrations ( $E C_{50}$ ) were in the low to medium $\mu \mathrm{M}$ range $\left(\mathrm{EC}_{50}<10 \mu \mathrm{M}\right.$ for flucythrinate, esfenvalerate, azinphos-methyl, propoxur; $\mathrm{EC}_{50}>10 \mu \mathrm{M}$ for tricaine). Modulation of movement activities in different phases of the PMR (i.e., "fingerprint") by neuroactive test compounds varied across concentrations, showing that mode of action-specific PMR fingerprints are also concentration-dependent. Above concentrations causing $10 \%$ lethality $\left(\mathrm{LC}_{10} ; 48 \mathrm{~h}\right), 3,4$-dichloroaniline caused movement inhibition. This substance presumably is not neuroactive; its effect on the PMR therefore is considered a secondary toxic effect. Quantitative morphological examinations of chemically exposed embryos showed that malformations occurred only above PMR effect concentrations, indicating that changes in the PMR were not due to such indirect effects. The PMR assay will provide a useful measure in ecotoxicological risk assessment of neuroactive chemicals with zebrafish embryos and could potentially be used to infer acute fish toxicity levels from PMR effect concentrations of neurotoxic compounds.
\end{abstract}

\section{Introduction}

Acute fish toxicity (AFT) tests are the most frequently used vertebrate tests in regulatory ecotoxicology (Scholz et al., 2013). They are typically conducted according to the standard test guideline OECD TG 203 (OECD, 2019) or similar guidelines. Fish embryo toxicity (FET) tests are proposed as alternatives to AFT tests for animal welfare reasons (Embry et al., 2010; Nagel, 2002; Scholz et al., 2008; Strähle et al., 2012), and OECD TG 236 has been established for chemical toxicity testing with the FET test (Busquet et al., 2014; OECD, 2013).

The sensitivities of the FET and AFT tests are generally similar (Belanger et al., 2013; Lammer et al., 2009a); however, particularly neurotoxic substances were found to be considerably less acutely toxic to fish embryos than to adult fish (Knöbel et al., 2012; Klüver et al., 2015; Sobanska et al., 2018). Failure of the respiratory system is considered to be the cause of neurotoxic chemical-induced lethality in adult fish (Bradbury et al., 2008; Russom et al., 2014). However, for fish embryos, respiratory failure does not appear to be caused by neurotoxic chemicals, since gas exchange and distribution mainly proceed via passive diffusion at those life stages. The function of the gills and the cardiovascular system is not required for distribution of oxygen in embryo stages unlike in adult fish (Jacob et al., 2002; Rombough, 2002). Interestingly, however, concentrations of neuroactive compounds causing lethality in adult fish were found to cause changes in locomotion of embryos; these may thus be used as indicators of concentrations of neuroactive compounds that would be acutely toxic for adult fish (Klüver et al., 2015).

Behavior-based biotests with zebrafish embryos enable detection of the neuroactive potential of chemicals (refer to review articles by Legradi et al., 2015; d'Amora and Giordani, 2018; Basnet
Received April 2, 2020; Accepted July 23, 2021; Epub August 2, 2021; ( ) The Authors, 2022.

ALTEX 39(1), 082-094. doi:10.14573/altex.2004021

Stefan Scholz, PhD; Till Luckenbach, PhD

Department Bioanalytical Ecotoxicology

Helmholtz Centre for Environmental Research (UFZ)

Permoserstr. 15, 04318 Leipzig, Germany

(stefan.scholz@ufz.de; till.luckenbach@ufz.de)
This is an Open Access article distributed under the terms of the Creative Commons Attribution 4.0 International license (http://creativecommons.org/licenses/by/4.0/), which permits unrestricted use, distribution and reproduction in any medium, provided the original work is appropriately cited. 
et al., 2019; Ogungbemi et al., 2019). One is the so-called photomotor response (PMR) measurement. It quantifies the undirected, 5-7 s long movement of zebrafish embryos at 30-35 hours post fertilization (hpf) elicited by a short light pulse. The PMR is mediated by stimulation of the opsin-based photoreceptors in the hindbrain by a light pulse. Neuroactive compounds were found to modulate the PMR (e.g., Kokel et al., 2010, 2013a,b; Copmans et al., 2016; Gauthier and Vijayan, 2018). During PMR measurements, embryos are kept in the dark and are exposed to two short light pulses, typically after $10 \mathrm{~s}$ and $20 \mathrm{~s}$. The 30 -s PMR measurement can be subdivided into four main phases: The background phase (PRE; 0-10 s) during which spontaneous movement of the embryos can occur is prior to the first light pulse; the latency phase $(\mathrm{L} ; \sim 1 \mathrm{~s})$ is defined as the phase between the first light pulse and the behavioral response; in the excitation phase (E; $9 \mathrm{~s})$, embryos show a behavioral response to the first light pulse; the second light pulse is followed by the refractory $(\mathrm{R})$ phase $(10 \mathrm{~s})$, during which the embryos are insensitive to any further light stimuli. In each of the PMR phases, the movement characteristics of embryos exposed to neuroactive chemicals can differ from those of untreated control embryos. The movement activity can be enhanced or reduced in the excitation phase, and it can be prolonged or delayed and extended into the refractory phase. The second light pulse may trigger movements of embryos exposed to certain neuroactive compounds. Spontaneous movements of the embryo can be enhanced during the background and evoked during the refractory phases (Reif et al., 2016; Kokel et al., 2010; Copmans et al., 2016). The embryonal movement activity, represented by the so-called "motion index", is quantified by determining the number of pixels that change due to movements of the embryo in the well within subsequent video frames. The phase-resolved analysis of the motion index has been used to establish fingerprints enabling compound classification (Kokel et al., 2010; Copmans et al., 2016).

The quantitative description of concentration-dependent effects of the entire PMR measurement is challenging for various reasons: 1) The activity parameter data is highly variable and not normally distributed; 2) the PMR effect is complex; chemically caused shifts of events on the PMR timeline were so far not included in effect quantification; 3) effect characteristics can be inconsistent across concentrations as, e.g., shown for tributyltin chloride (Reif et al., 2016). Therefore, PMR data analysis was previously often limited to a qualitative description of differences between control and exposure groups (Reif et al., 2016; Legradi et al., 2015).

In this study, PMR data for zebrafish embryos were obtained for four known neuroactive pesticides, the organophosphate azinphos-methyl, the carbamate propoxur (Fukuto, 1990; Gruber and Munn, 1998) and the $\alpha$-cyano-pyrethroids esfenvalerate and flucythrinate (Fukuto, 1990; Soderlund and Bloomquist, 1989; Attili and Hughes, 2014; Gruber and Munn, 1998). Furthermore, the effect of tricaine on the PMR was examined as a representative compound with an anesthetic mode of action (MoA; Attili and Hughes, 2014).

The zebrafish embryos at the stage used in the experiments ( $\sim 35 \mathrm{hpf}$ ) possess molecular targets for neuroactive compounds. For instance, acetylcholine esterase $(\mathrm{AChE})$ is present in prima- ry motor neurons from $14 \mathrm{hpf}$ onwards and appears in sensory neurons and interneurons at around $24 \mathrm{hpf}$ (Hanneman and Westerfield, 1989). Furthermore, voltage-gated sodium channels are expressed and functional already in early developmental stages of zebrafish, indicated by the occurrence of spontaneous contractions at 17-30 hpf and sodium currents at muscle fibers in embryos (Buckingham and Ali, 2004; Coutts et al., 2006).

In this study, a data analysis approach was adapted that is based on comparing the distribution of movement activities of individual embryos from different treatments on the time axis. We show that by including the parameter time in the data analysis, a quantitative description of chemically elicited PMR concentration-effect analyses can be made. So far, a similar approach has only been applied in studies examining the locomotor response (LMR), another behavior-based biotest that measures the locomotion of zebrafish embryos, i.e., the distance covered within a certain time (Selderslaghs et al., 2010, 2013; Klüver et al., 2015). In contrast to the previous approach for LMR analysis (Selderslaghs et al., 2010), we included the parameter time as a second dimension and calculated a density volume of the time-dependent activity parameter. The difference in density volume between control and treatment was used for the quantitative assessment of the effect of chemical exposure on the PMR. We show that this data analysis approach improves the estimation of concentration-effect relationships for the PMR. We also investigated whether the PMR response patterns to the selected compounds were consistent across the tested concentration ranges. The effect of the non-neuroactive compound 3,4-dichloroaniline on the PMR was examined to determine to which extent the PMR is specific for neuroactive compounds. The PMR effect concentrations were set in relation to FET and AFT effect concentrations in order to obtain a measure for the sensitivity of the PMR. Lethal effect concentrations for adult or juvenile zebrafish were not available for all test compounds. Therefore, in order to avoid additional animal experiments with adult zebrafish and given the overall high correlation of fathead minnow with zebrafish FET data (Belanger et al., 2013), the respective AFT data for fathead minnow (Pimephales promelas) were used for comparison of AFT and PMR effect data.

\section{Animals, materials and methods}

\subsection{Chemicals and experimental solutions}

3,4-dichloroaniline (CAS RN 95-76-1, 99.8\% purity), tricaine (MS222; CAS RN 886-86-2, 98\% purity), propoxur (CAS RN 114-26-1, 99.8\% purity), azinphos-methyl (CAS RN 86-50-0, > 99\% purity), esfenvalerate (CAS RN 66230-04-4, 99.5\% purity), and flucythrinate (CAS RN 70124-77-5, analytical standard) were obtained from Sigma Aldrich (Deisenhofen, Germany). Exposure solutions were prepared by dilution of chemicals in reconstituted water made according to OECD TG 236 (OECD, 2013). All compounds exhibit no or a low degree of dissociation, as indicated by their $\mathrm{pKa}$ values. Hence, all stock solutions (equal to the highest compound concentrations tested) were at $\mathrm{pH} 7 \pm 1$. No deviation of the $\mathrm{pH}$ in the unbuffered exposure medium was 
observed at the end of exposures (measured in the treatments with the highest test compound concentrations with no mortality of embryos). The stock solutions were set up one day before start of the exposures in closed graduated glass flasks and were stirred for approximately $14 \mathrm{~h}$. The experimental solutions were prepared by serial dilutions of stock solutions. The stability of the concentrations of azinphos-methyl, propoxur, tricaine and 3,4-dichloroaniline in experimental solutions was examined in independent experiments by applying UV/VIS-absorption measurements (refer to Text $\mathrm{S}^{1}$ in the supplementary file for details).

Within $48 \mathrm{~h}$, a decline in concentrations of $<1 \%$ (azinphos-methyl, tricaine), $<5 \%$ (propoxur), and $<15 \%$ (3,4-dichloroaniline) from initial concentrations in the aqueous solutions was observed. It can therefore be assumed that the nominal concentrations of the compounds reported here concur with their actual concentrations to a high degree. For the pyrethroids esfenvalerate and flucythrinate, the exposure concentrations were below the detection limits for UV/VIS analysis. For esfenvalerate, a strong deviation from nominal concentrations and a rapid concentration decline during $24 \mathrm{~h}$ exposure has been observed previously in a semistatic exposure setup (Klüver et al., 2015). As structure and physico-chemical properties of flucythrinate are similar to esfenvalerate, a strong deviation of actual from nominal concentrations in the exposure medium is likely also for this compound, and the calculated effect concentrations for these compounds may therefore be overestimated. Oxygen saturation of exposure solutions was $>90 \%$.

\subsection{Maintenance of zebrafish, determination of zebrafish embryo toxicity (FET), and source of fathead minnow acute fish toxicity (AFT) data}

Information on zebrafish maintenance, embryo collection, exposure conditions and FET tests are provided in Text S2, Tab. $\mathrm{S} 3$ and Fig. S5 ${ }^{1}$. Exposures of embryos were from 2-96 hpf, and mortality data for $\mathrm{LC}_{50}$ (50\% lethal concentration) estimation at $48 \mathrm{~h}$ and $96 \mathrm{~h}$ were recorded.

AFT $\mathrm{LC}_{50}(96 \mathrm{~h})$ values for fathead minnow (Pimephales promelas) were retrieved from a database from the U.S. Environmental Protection Agency (US EPA, Russom et al., 1997) (see also Text S2, Retrieval of AFT data $\left.\left(\mathrm{LC}_{50}\right)^{1}\right)$.

\subsection{Photomotor response (PMR) assay}

Zebrafish embryos were exposed to concentration series of the model compounds (3,4-dichloroaniline, azinphos-methyl, propoxur, tricaine) with the zebrafish FET $\mathrm{LC}_{10}(96 \mathrm{~h})$ as highest test concentration. For compounds not causing lethal effects in the FET test, the highest test concentration corresponded to maximum water solubility (flucythrinate, esfenvalerate; refer to Tab. $\mathrm{S}^{1}{ }^{1}$ for an overview of all test concentrations for each test compound). Embryos were exposed in crystallizing dishes from 2 to $\sim 30-35 \mathrm{hpf}$ at $26^{\circ} \mathrm{C}$. Embryos at this stage were then subjected to PMR measurements. The time of day when embryos were at 30-35 hpf and the PMR measurements were performed corresponded to 2-7 pm. Prior to PMR measurements, embryos were manually dechorionated with two forceps. The dechorionated embryos were transferred together with $400 \mu \mathrm{L}$ of the exposure solution to the 24 central, round-shaped wells of a 96-well plate (Cellstar Greiner Bio-One, Frickenhausen, Germany). The zoom-lens of the camera used to video-record the embryos was adjusted to these central 24 wells, providing sufficient magnification to resolve the movement of the embryos while enabling simultaneous recordings of multiple wells. Five embryos were placed in each well, and six wells were used per control/treatment. Three treatments along with a control were run simultaneously in each experiment. To obtain a measure for the variability across controls from different plates and from different positions on a plate, an experiment with three control plates (control embryos in all 24 wells) was performed. Plates were kept in the dark at $28^{\circ} \mathrm{C}$ for at least $15 \mathrm{~min}$ before PMR measurements were started. Prior to the measurement, the exposure solution level in each well was adjusted to the maximum possible volume by addition of the respective exposure solution to avoid formation of a meniscus. PMR measurements were performed using a ZebraBox (ViewPoint, Lyon, France) set to quantification mode, transparent detection, and a sensitivity of 3 , using 25 video frames per second. The resolution was $0.2 \mathrm{~mm} /$ pixel. The temperature of the exposure solutions was maintained at $28^{\circ} \mathrm{C}$ prior to and during the measurements by placing the multiwell plate with the embryos in a waterbath. The embryos were recorded for $30 \mathrm{~s}$ using infrared (IR) illumination and an IR sensitive camera. At $10 \mathrm{~s}$ and at $20 \mathrm{~s}$ of the measurement, a $1 \mathrm{~s}$ pulse of visible light was emitted from the top light array in the ZebraBox (top light set to maximum intensity determined as 69384 lux using a LI-COR radiation sensor, Bad Homburg, Germany). Embryo movements are expressed by motion index values calculated by the ViewPoint software based on the numbers of altered grey values per pixel and per video frame. For further analysis, motion index values were exported from the software as a text file.

\subsection{PMR data analysis and statistics}

Data analysis was performed using the software $\mathrm{R}^{2}$. For PMR effect quantification and calculations for qualitative PMR effect assessment (fingerprint), we developed the program "PMaveRicks" (available on request), which consists of $\mathrm{R}$ applets organized in $\mathrm{R}$ AnalyticFlow ${ }^{3}$. Prior to analysis, motion index values from the 6 wells of the control/each treatment per plate were pooled and then increased by one unit to enable ln-transformation of values of 0 ; motion index values were then $\ln$-transformed.

\section{Quantitative analysis of the PMR for concentration-}

effect modeling

For quantification of PMR effects based on the so-called two-dimensional (2D) density approach, a 2D kernel density function was fitted to the motion index values of each treatment/control

\footnotetext{
1 doi:10.14573/altex.2004021s

2 http://www.R-project.org/

3 http://r.analyticflow.com/en/
} 
from the 5-25 s period of the PMR measurement. Densities were estimated using the kde2d function within the R-package MASS (Venables and Ripley, 2002). The number of grid points (n) was set to 100 in both directions, and the limit of the rectangle covered by the grid was set to $0,30,-1,10$. The output of the function is a matrix of density values with the two dimensions motion index values and time. The calculated density describes the distribution of the motion index values along the time axis, and the density integral equals a volume of 1 . The density matrix calculated for the respective plate control was subtracted from the density matrix for a treatment; the resulting absolute value composes the effect matrix. The integral of the effect matrix equals the effect density volume, which defines the probability for a treatment being different from the control. The integral of the effect density is scaled between 0 and 1 and is used as the final PMR effect parameter.

Three control plates were measured to estimate variations between controls. In each plate, embryos in the wells of the top row were considered as reference to which the PMR 2D-density values of the three following rows were compared. Across all plates, the mean difference between 2D-density values for these subsets of control embryos was $19 \%$ (coefficient of variation was $4 \%$; a mean density difference of $0 \%$ would represent a $100 \%$ overlap of density surfaces). Based on the assumption that PMR effects can be described by a sigmoidal regression model, concentration response curves were fitted using a four parametric logistic model (see Text $\mathrm{S} 2$, Equation for concentration response modeling ${ }^{1}$ ) with a minimum difference in density fixed to $19 \%$ (see paragraph "Concentration dependency of PMR patterns" below). For esfenvalerate, no clear PMR effect saturation was seen (Fig. 2d). However, this was considered to impact the calculation of effect concentrations only negligibly, since the maximum effects of this compound were in the range of $60 \%$, which was similar to effect saturation levels of the other test compounds. The time course of changes of the motion index was illustrated with a loess spline fitted to motion index values of each treatment using the R-function "loess".

Furthermore, for PMR effect parameter estimation, the overlapping area (OA) approach was applied. This approach has previously been used for LMR analysis (Selderslaghs et al., 2010). OA-density estimation was performed using the bckden function of the R package evmix. The bandwidth was set to 0.5 . In order to avoid density estimations for motion index values of less than 0 , we used a boundary-corrected kernel estimator. Motion index values from the $5-25 \mathrm{~s}$ period were used. The resulting density describes the distribution of the motion index values. The area under the density curve equals 1 . The overlapping areas of the density curves of the treatments and the corresponding control were calculated. The resulting value is scaled to a value between 0 and 1, describing the differences between the density curves; the PMR effect parameter is defined by one minus the overlapping area. Based on the data from the three multiwell plates containing only controls (see above), the mean difference in OA densities across controls was determined, which was $7 \% \pm 3.7$ (SD). Details on PMR effect modelling based on density volumes and OA are given in Text S4, Tab. S2, Figs. S3, S41.

\section{Concentration dependency of PMR patterns}

To group chemical concentrations that cause similar PMR movement patterns, a cluster analysis was performed. Prior to the analysis, benchmark concentrations (BMC) were estimated using the software BMDS2504. Concentrations below BMC were considered to be no-effect concentrations and were excluded from the cluster analysis. For each experiment, motion index values of each treatment were pooled with all detected values of specific time intervals (PRE: 0-10 s, L: 10-11 s, E1: 11-12 s, E2: 12-16 s, E3: $16-20 \mathrm{~s}, \mathrm{R} 1: 20-22 \mathrm{~s}, \mathrm{R} 2: 22-30 \mathrm{~s}$ ), and the $75 \%(\mathrm{Q} 3)$ and the $25 \%(\mathrm{Q} 1)$ quartiles were calculated as described by Kokel et al. (2010). The quartiles were normalized to the corresponding quartile of the plate control. The normalized quartiles of a treatment represent the barcode-like PMR movement patterns (fingerprints). Fingerprint replicates of a chemical concentration were summarized by calculating the mean of each quartile. Hierarchical clustering with complete linkage and a Euclidean distance matrix was performed using the MeV 2.0 software, a component of the TM4 package (Saeed et al., 2003).

\subsection{Quantitative assessment of morphological changes by chemical exposure}

Investigations of chemical effects on embryo morphology served to obtain an indication whether a change in the PMR response is related to morphological alterations and/or developmental delay. Upon exposure to chemicals, the morphology of zebrafish embryos at 30 hpf was examined using a quantitative approach based on image analysis with the software FishInspector. The embryos that were morphologically assessed were at the same developmental stage as the embryos subjected to the PMR measurements. Embryos from treatments with two different concentrations of test chemicals were examined for changes in morphology. The concentrations corresponded to the PMR 50\% effect concentrations $\left(\mathrm{EC}_{50}\right)$ and to the FET 50\% lethal effect concentrations $\left(\mathrm{LC}_{50} ; 48 \mathrm{~h}\right)$. In the case that the chemical did not cause lethal effects in the FET test and no FET LC L $_{50}(48 \mathrm{~h})$ value could be determined, the chemical concentration corresponding to the maximum water solubility of the compound was used. Images of the zebrafish embryos were obtained and analyzed as described by Teixido et al. (2019). Prior to imaging, embryos at 24 to $30 \mathrm{hpf}$ were dechorionated using forceps. Dimension values of diverse morphological features (e.g., body length, eye size, yolk size, otolith-eye distance, body curvature) were compared between controls and exposed embryos. Overlapping standard deviations from three independent replicate experiments were considered to indicate non-significant differences in morphology.

\section{Results and discussion}

Behavioral assays with zebrafish embryos have been suggested as an approach for identifying chemicals with neuroactive MoAs (Kokel et al., 2010; Klüver et al., 2015) and to improve the prediction of AFT (Klüver et al., 2015). At present, analyses of PMR

\footnotetext{
4 Version 2.5 is no longer available. A recent version of the software with similar functionality is available at https://www.epa.gov/bmds/benchmark-dose-software-bmds-version-3
} 

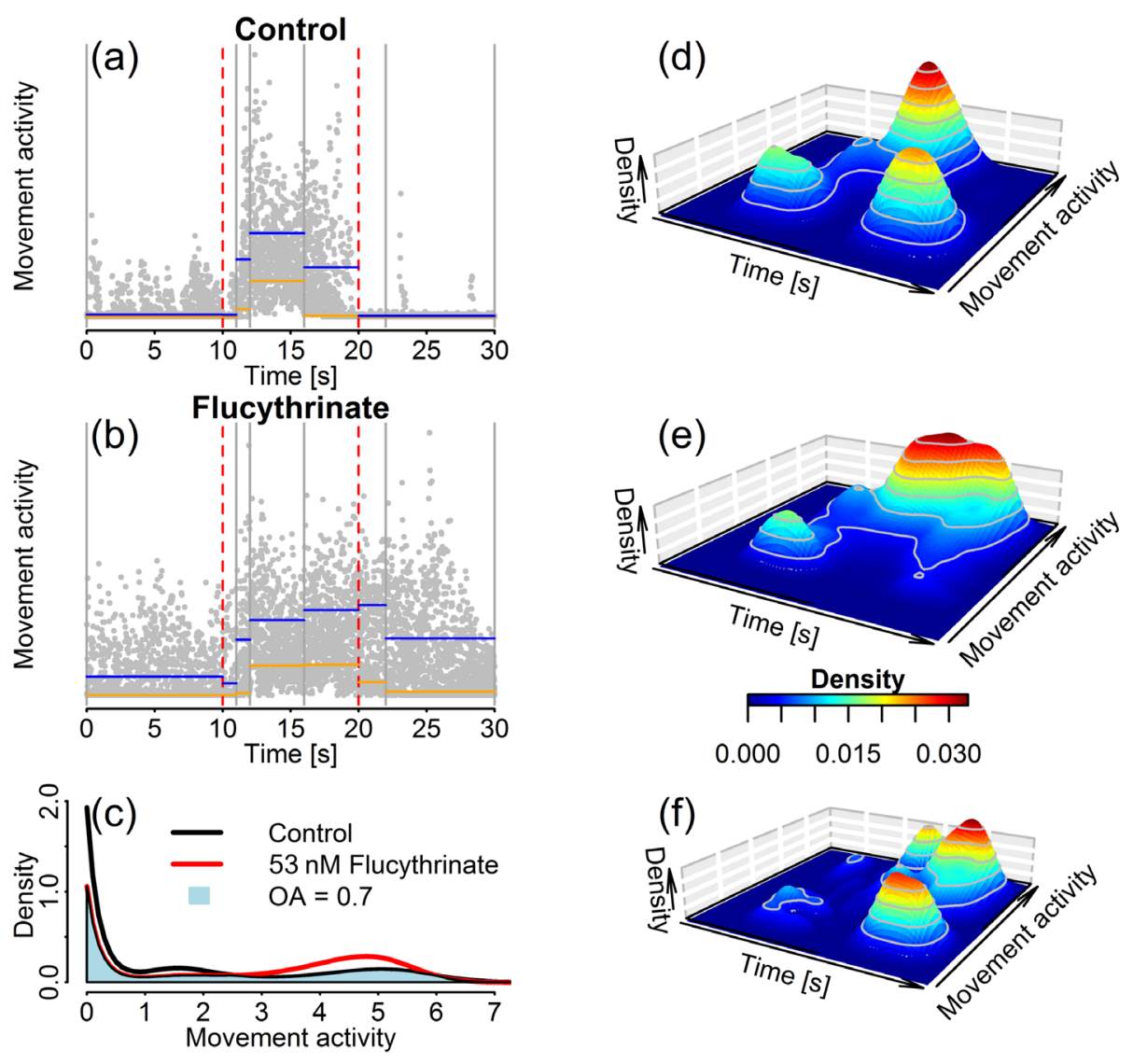

Fig. 1: Exemplary graphical illustrations of motion index data representing the movement activities of zebrafish embryos during a PMR measurement period (a,b), and graphs of PMR data analyzed with the OA-density approach (c) and the 2D-density approach (d,e,f).

Data are from control embryos and from embryos treated with the voltage-gated sodium channel agonist flucythrinate (53 nM). For further explanations of the OA- and 2D density approaches, please refer to the main text. (a) Control, (b) flucythrinate treatment: Each point in the graphs represents the motion index value for five embryos in a well at a given time point. Data for six simultaneously recorded wells per condition are shown. Blue and orange horizontal lines represent the upper and lower quartiles, respectively, of the motion index values from the different time phases of the PMR measurement (PRE, background; L, latency; E1, E2, E3, excitatory; R1, R2, refractory phases). The vertical dashed lines represent the time points of the $1 \mathrm{~s}$ light pulse. (c) OA-density approach: Density curves were fitted to the motion index values from the 5-25 s period of the PMR measurement for the control group (black line) and the flucythrinate treatment (red line), and the resulting OA between the density curves (lightblue area) is shown; integration of the OA equals the PMR effect $(\mathrm{OA}=0.7)$.

(d) Control, (e) flucythrinate treatment; 2D-density approach: Time-resolved densities were fitted to the motion index values from control and flucythrinate-treated embryos. $(\mathrm{OA}=0.4)$ (f) Graphical illustration of the differences in the time-resolved densities of the PMR movement activities between control and flucythrinate treatment obtained by subtraction of (d) and (e); integration of the density volume equals the final PMR effect.

and LMR represent the methods most frequently used to quantify behavioral effects in fish embryos (Ogungbemi et al., 2019). This study focused on the analysis of the PMR to address two main questions: (1) How can the time course of the PMR response be considered appropriately in the characterization and quantification of chemical effects? (2) Are PMR effect concentrations similar to AFT concentrations of neuroactive compounds, and could they be used to predict AFT?

\subsection{PMR effect quantification using a 2D kernel density function (2D-density approach)}

The time-dependent PMR movement patterns can be altered by neuroactive compounds in multiple ways. As demonstrated by Kokel et al. (2010), decreased and/or increased movement activity can occur in one or several of the four PMR phases (PRE, L, E, R). However, in some cases, neuroactive compounds cause changes in the time course of the PMR rather than in the level of 
movement activity. As an example for a shift of the PMR movement activity on the time axis, the PMR pattern recorded upon exposure of embryos to $53 \mathrm{nM}$ flucythrinate, a voltage-gated sodium agonist, is depicted in Figure 1. Compared to the controls (Fig. 1a), the movement response of flucythrinate-exposed embryos was extended into the refractory (R) phase (Fig. 1b). When using the OA-density approach for analysis of the movement activity parameter (Selderslaghs et al., 2010) for a PMR measurement, differences between treatment and control do not become visible because the changes in movement along the time axis are not considered (Fig. 1c). Considering the changes in movement along the time axis, however, appears to be important to be able to clearly differentiate between the PMR of individuals subjected to different treatments. When the PMR data for the test compounds were analyzed with the OA-density approach, only little difference between treatments was seen in most cases (refer to Fig. $\mathrm{S}^{1}{ }^{1}$ ). In order to describe the frequency of movements (i.e., changes in pixels) at a certain time point, we added time as a second dimension in our "2D-density approach", incorporating the scatter and time dependency of the PMR movement activity parameter (Figs. 1d (control), 1e (treatment)). Like for the OA-density analysis, integration of the 2D-density equals 1 . However, the value obtained with the 2D-density approach equals a volume along the three axes movement activity, time, and density; the value does not represent an area as it does in the OA-density analysis. The value obtained when subtracting the shared density volume from 1 indicates the degree of difference between the groups; the difference in volume is taken as an effect measure, which is scaled between 0 and 1 . The difference in density between the flucythrinate treatment and control is depicted in the graph in Figure 1f.

\subsection{Concentration-dependent PMR effects}

Analysis of the 2D-density differences indicated that the similarity of controls never approached $0 \%$, which would represent a $100 \%$ overlap of the density surfaces. This can be explained by an intrinsic variability of the movement patterns, preventing a $100 \%$ match of density surfaces between replicated experiments. A comparison across control replicates determined with three multi-well plates containing only control embryos indicated an average difference of $19 \%$, which is similar to the mean variation observed for LMR data of zebrafish embryos quantified with the OA approach (Selderslaghs et al., 2010). Therefore, for concentration-response modeling, the minimum was set to 19\% (Fig. 2, Tab. 1, see also above). The residual standard errors in the non-linear concentration-effect regression analyses ranged from $6 \%$ for propoxur to $10 \%$ for tricaine. Residual standard errors were not found to be concentration-dependent and did not differ in replicate experiments (for further details refer to Text S2 ${ }^{1}$ ), confirming that the logistic model was well-suited to describe the PMR effects. To determine the variability of controls across different plates, the PMR values from control embryos from 62 plates were evaluated. The motion index values of controls varied by a factor of up to 10 between plates. Motion index values for each embryo on a plate were therefore normalized to the respective controls on this plate before values from different plates were combined for further analysis (Text S4, Tab. S2, Fig. S3, S4 ${ }^{1}$ ).

A maximum PMR effect of $100 \%$ would represent a complete separation of the density volumes between a treatment and the control. PMR effects by test compounds in our experiments were between $6-90 \%$ (Fig. 2), indicating that density volumes of controls and treatments were jointly shared to a certain degree in all cases. For example, $77 \mu \mathrm{M}$ tricaine caused a general movement inhibition in the excitation phase, but tricaine-treated and control embryos showed the same movement activity during latency, background, and refractory phases (Fig. 3, for further details see Text S3, Fig. S2 ${ }^{1}$ ). This results in an equal density distribution in those three phases; differences are seen only in the excitation phase. Based on the assumption that saturation of the effect occurs at a certain point and a modeled saturation level, the maximum effects of the examined compounds were $\sim 86 \%$ for tricaine, $\sim 66 \%$ for flucythrinate, $55 \%$ for esfenvalerate, $\sim 47 \%$ for azinphos-methyl, and 39\% for propoxur (Fig. 2, Tab. 1).

These differences in maximum effects can be related to compound-specific alterations of the PMR that appear to correspond to the neuroactive MoAs: The complete inhibition of movement in the excitatory phase by the anesthetic tricaine is probably related to its MoA as voltage-gated sodium channel antagonist (Attili and Hughes, 2014). In contrast, flucythrinate and esfenvalerate were shown to act as voltage-gated sodium channel agonists, activating the voltage-gated sodium channels to remain in an open state (Soderlund and Bloomquist, 1989); they caused a prolonged movement extended into the refractory phase and reduced movement activity in the excitatory phase. Azinphos-methyl and propoxur, inhibitors of AChE (Gruber and Munn, 1998), caused increased movement during the excitation phase with prolongation into the refractory phase (Fig. 3, for further details see Tab. S4 ${ }^{1}$ ).

The compound concentration at which the maximum PMR effect is seen may indicate the concentration at which target saturation occurs. PMR effects by the neuroactive test compounds were seen at concentrations in the sublethal range ( $<\mathrm{LC}_{10} ; 48 \mathrm{~h}$ ), whereas PMR effects by non-neuroactive 3,4dichloroaniline occurred only in the lethal concentration range of the compound at concentrations $>$ FET LC $\mathrm{L}_{10}(48 \mathrm{~h})$, showing a $35 \%$ PMR effect at the highest test concentration (Fig. 2). The PMR effects by the neuroactive compounds azinphos-methyl, propoxur and tricaine at concentrations in the range of the $\mathrm{LC}_{10}$ $(48 \mathrm{~h})$ and above differed from those found at lower concentrations (Tab. S4 ${ }^{1}$ ). A general movement inhibition during the excitation phase was seen for the compounds when the exposure concentrations were $\geq$ FET LC $_{10}(48 \mathrm{~h})$; PMR effect data for these concentrations were therefore excluded from concentration-effect analysis. The PMR effects of high concentrations of 3,4-dichloroaniline were in the same order of magnitude of the FET $\mathrm{LC}_{10}(48 \mathrm{~h})$ and above. PMR effects by chemicals at concentrations $\geq$ FET LC $_{10}(48 \mathrm{~h})$ likely represent unspecific effects rather than specific neuroactive interaction; the FET $\mathrm{LC}_{10}$ $(48 \mathrm{~h})$ therefore appears to be suitable as a threshold defining the concentration range causing predominantly specific neuroactive PMR effects. 

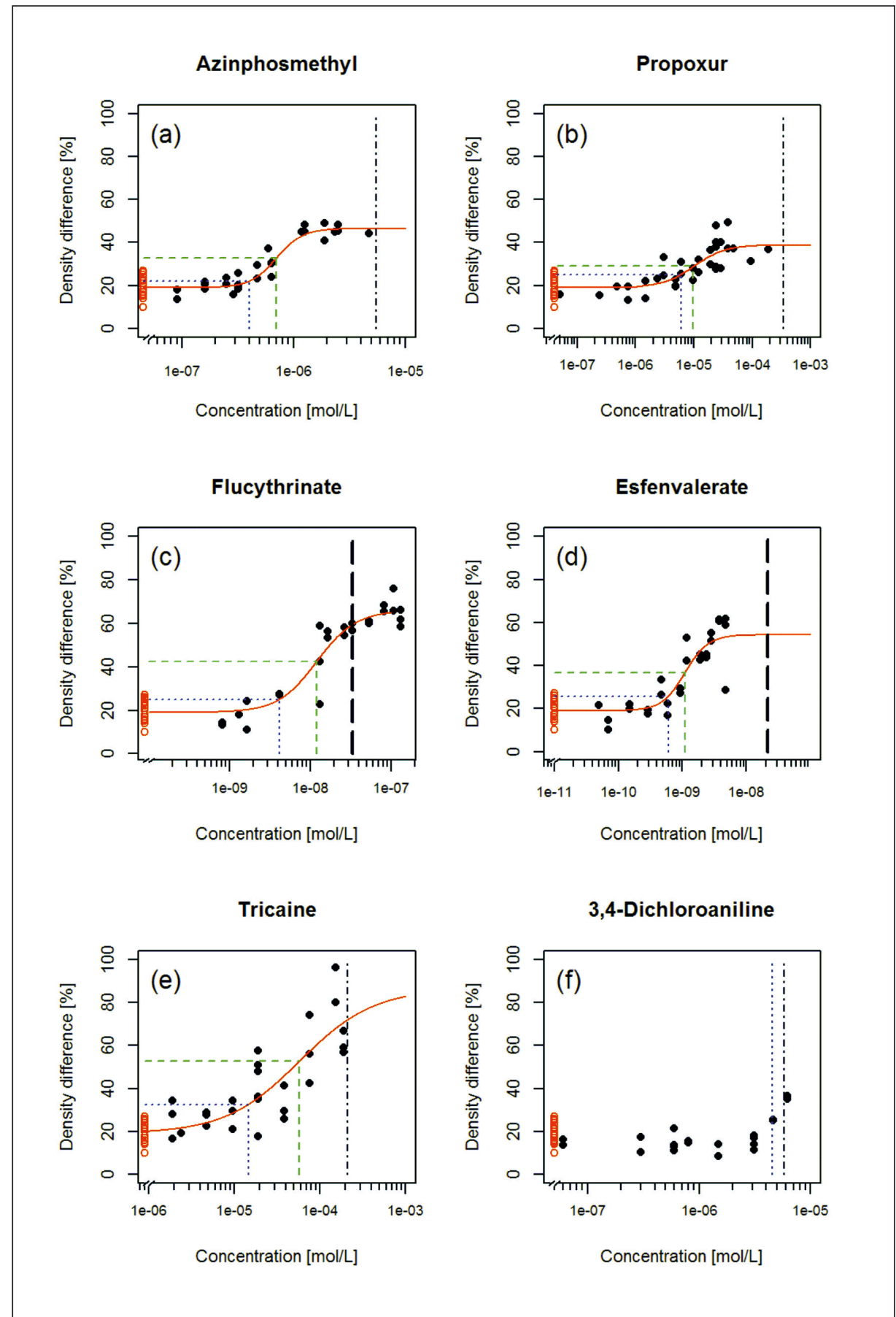

Fig. 2: Concentration-dependent PMR in zebrafish embryos (35 hpf) determined for the AChE inhibitors azinphosmethyl (a) and propoxur (b); the voltage-gated sodium channel agonists flucythrinate (c) and esfenvalerate (d); the voltage-gated sodium channel antagonist tricaine (e); and the non-neuroactive reference compound 3,4-dichloroaniline (f) Data points (filled black circles) represent differences in the timeresolved densities of the activity parameters between control and exposed embryos (2D-density approach). An effect of $0 \%$ and $100 \%$ would refer to complete overlap or separation, respectively, of the density volumes. The control variability of the PMR is indicated by open red circles. A logistic model (Eq. 1, Section S2 ${ }^{1}$ ) was fitted to the data and used to calculate $\mathrm{EC}_{50}$ values; benchmark concentrations (BMC) were estimated with BMDS250. Vertical lines indicate FET LC 10 (48 h) (dash-dot) and baseline toxicity (dotted) or maximum water solubility (dashed) of compounds for comparison.
Basically, changes in the PMR at sublethal chemical concentrations can also be due to non-neuroactive effects, such as morphological alterations. Such a connection of behavioral responses with gross morphological alterations has been shown in a meta-analysis of zebrafish developmental toxicity data (Ducharme et al., 2013). However, in our study no association of a modified PMR with morphological alterations was seen. A quantitative image-based assessment of phenotypes indicated morpho- logical effects by the test chemicals only at high concentrations that were close to lethal (refer to Text S5, Tab. S6 ${ }^{1}$ ).

Regarding neuroactive potencies based on PMR EC 50 values, the test compounds were ranked as follows: esfenvalerate $<$ flucythrinate $<<$ azinphos-methyl $<$ propoxur $<$ tricaine (Tab. 1). With $\mathrm{EC}_{50}$ values in the low $\mathrm{nM}$ range, the voltage-gated sodium channel agonists esfenvalerate and flucythrinate represent the compounds with the highest potency to exert effects on the PMR, 


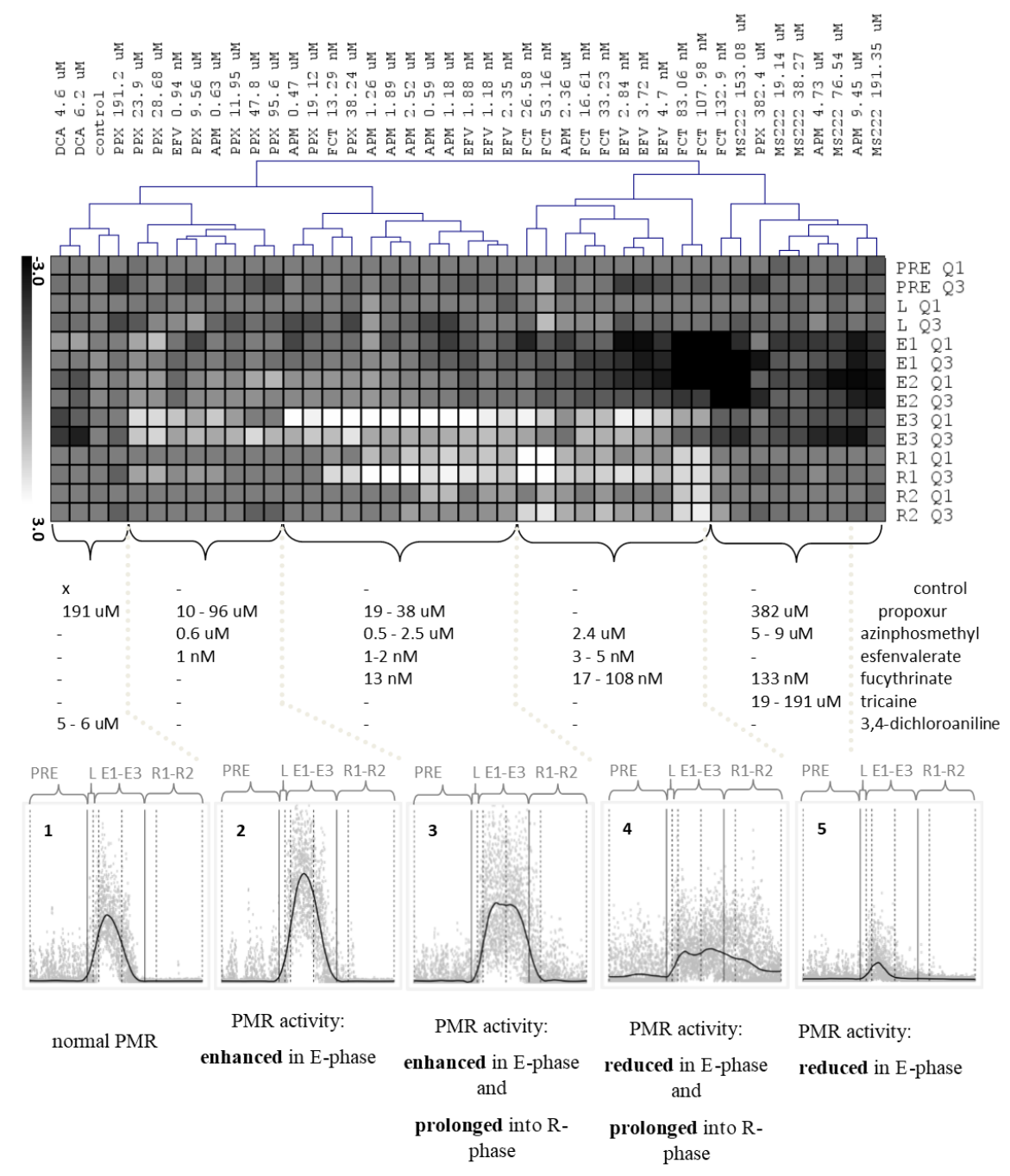

Fig. 3: Cluster analysis of PMR fingerprints determined in zebrafish embryos from chemical treatments and a control

The PMR fingerprints are visualized in a heatmap with movement activities scaled from -3 to 3 depicting the first (Q1) and the third quartile (Q3) of PMR subphases (PRE, background phase; L, latency phase; E, excitatory phase; R, refractory phase). The five main clusters to which fingerprints could be allocated are illustrated by representative graphs with single data points and a time-dependent spline of the normal-scaled motion index: Cluster 1, control; cluster 2, $0.63 \mu \mathrm{M}$ azinphos-methyl; cluster 3, $2.5 \mu \mathrm{M}$ azinphos-methyl; cluster 4, $53 \mathrm{nM}$ flucythrinate; cluster 5, 4.7 $\mu \mathrm{M}$ azinphos-methyl. Substance and concentration ranges of the main clusters are shown. Concentration ranges of test compounds allocated to respective clusters are also indicated. APM, azinphos-methyl; DCA, 3,4-dichloroaniline; EFV, esfenvalerate; FCT, flucythrinate; MS222, tricaine; PPX, propoxur

whereas the $\mathrm{EC}_{50}$ for the least potent compound, the voltage-gated sodium channel antagonist tricaine, was four orders of magnitude higher (Tab. 1). Of the tested acetylcholinesterase inhibitors, the $\mathrm{EC}_{50}$ for the organophosphate azinphos-methyl was approximately 40 times lower than that for the carbamate propoxur (Tab. 1), which corresponds to the differences in the bimolecular reaction constants for $\mathrm{AChE}$ binding of both compounds or their active transformation products $\left(1 \times 10^{6} \mathrm{~L} \mathrm{x} \mathrm{mol}^{-1} \mathrm{x} \mathrm{min}^{-1}\right.$ for az- inphos-methyl oxon and $0.13 \times 10^{6} \mathrm{~L} \mathrm{x} \mathrm{mol}^{-1} \mathrm{x} \mathrm{min}^{-1}$ for propoxur, AChE from electric eel (Herzsprung et al., 1992)). In contrast to carbamates, organophosphates require metabolic activation by cytochrome P450 enzymes, resulting in the oxon-metabolite that acts as an AChE inhibitor (de Brujin et al., 1993). The strong PMR effect of azinphos-methyl (Fig. 2, 3; Tab. 1; Tab. S4, S5 ${ }^{1}$ ) thus indicates that the zebrafish embryo at this developmental stage is already able to metabolically activate organophosphates; indeed, 
evidence for metabolization of xenobiotics in the respective zebrafish embryo stages has been reported (Brox et al., 2016).

As shown here for compounds with different neuroactive MoAs, PMR effect parameter values obtained with the 2D-density approach incorporating the parameters motion index and time could successfully be used for concentration-PMR effect modeling, enabling PMR effect quantification. In contrast, PMR effect parameter values calculated with the OA approach showed a weaker or no clear concentration dependence (Text S4, Tab. S2, Fig. S3, S4 ${ }^{1}$ ).

\subsection{Comparing PMR effects and lethal effects in zebrafish embryos and adult fish (fathead minnow)}

Due to a rapid decline in concentrations in the exposure solutions during the experiment, the PMR effect concentrations, as determined here, could be overestimated, specifically for the hydrophobic pyrethroids esfenvalerate and flucythrinate (Klüver et al., 2015). This may explain the relatively small difference of the PMR effect and baseline toxicity concentrations observed particularly for flucythrinate. Adaptation of the exposure protocol for FET and PMR analysis would be required to retrieve a stable exposure concentration, for instance by using a flow-through system (Lammer et al., 2009b) and to demonstrate that PMR effect concentrations are well below baseline toxicity. Therefore, the comparison of the effect concentrations at this stage must be regarded as preliminary. However, the observation of PMR effects at sublethal concentrations confirms the diagnostic capacity of the PMR for detection of potential neuroactive compounds.

Behavioral assays with fish embryos have been suggested earlier as an approach for predicting acute toxicity of neuroactive compounds for adult fish (Klüver et al., 2015). For determining whether effect concentrations of chemicals on PMR of fish embryos correspond with lethal effect concentrations in adult fish, PMR effect concentrations were compared with zebrafish FET $\mathrm{LC}_{50}$ and fathead minnow (Pimephales promelas) AFT data (Tab. 1, Fig. 4). Although it can be assumed that the fathead minnow AFT data provide a good representation of such data for zebrafish (refer to Belanger et al., 2013), discrepancies between AFT data for fathead minnow and zebrafish cannot be fully excluded.

The FET $\mathrm{LC}_{50}$ values for azinphos-methyl, propoxur, flucythrinate, esfenvalerate and 3,4-dichloroaniline were determined for $48 \mathrm{~h}$ and $96 \mathrm{~h}$ exposure durations. Except for flucythrinate and esfenvalerate (both compounds did not cause mortality in FET tests), $\mathrm{LC}_{50}$ values after $48 \mathrm{~h}$ and $96 \mathrm{~h}$ exposure durations were similar (Tab. 1; for further details regarding the FET results, refer to Text $\mathrm{S} 2{ }^{1}$ for Eq. 1 used for concentration-response modeling and to Tab. S3 and Fig. S5 ${ }^{1}$ for concentration-response curves and modeling parameters).

For the neuroactive substances azinphos-methyl, propoxur, flucythrinate and esfenvalerate, FET LC $\mathrm{L}_{50}(48 \mathrm{~h})$ values exceeded the AFT LC $_{50}(96 \mathrm{~h})$ values by 12 - to 66 -fold or compounds did not cause lethal embryotoxic effects (Tab. 1). Hence, FET

Tab. 1: Names, Chemical Abstract Service (CAS) numbers, log $\mathrm{K}_{\mathrm{ow}}$ values, water solubilities, acute fish and fish embryo toxicities (AFT, FET), and photomotor response (PMR) effect concentrations of test compounds

Azinphos-methyl, propoxur, flucythrinate, esfenvalerate and tricaine were applied as neuroactive test compounds; 3,4-dichloroaniline is not neuroactive. AFT LC 50 (96 h; fathead minnow) data were retrieved from the literature, FET LC 50 (48 h) data are from this study. Baseline toxicities of compounds to zebrafish embryos were calculated based on their log $\mathrm{K}_{\mathrm{ow}}$ (Klüver et al., 2016). PMR effects were estimated with the 2D-density approach, and concentration-dependencies of effects were modelled using a 4-parameter logistic regression with the minimum effect set to $19 \%$ (for further details refer to text; regression equation: Eq. 1 in Text S2 ${ }^{1}$ ). PMR EC 50 values are shown together with the estimated parameters slope and max-min, representing the effect range. PMR effect benchmark concentrations (BMC) were estimated with BDMS software. The corresponding PMR effects at BMC are given as $\mathrm{x}(\mathrm{ECx})$.

\begin{tabular}{|c|c|c|c|c|c|c|c|c|c|c|c|c|}
\hline & \multirow{2}{*}{\multicolumn{3}{|c|}{\begin{tabular}{|l|l|} 
AFT & FET \\
\end{tabular}}} & \multirow{2}{*}{\multicolumn{6}{|c|}{ Fish embryo behavioral effects - PMR at $35 \mathrm{hpf}$}} \\
\hline & & & & & & & & & & & & \\
\hline Substance & CAS \# & $\log _{\mathrm{Low}}{ }$ & $\begin{array}{l}\text { Water } \\
\text { solubility }\end{array}$ & $\begin{array}{l}\text { LC50 } \\
(96 h)^{b}\end{array}$ & $\begin{array}{l}\text { LC50 } \\
(48 \mathrm{~h})\end{array}$ & $\begin{array}{l}\text { Baseline } \\
\text { toxicity }^{c}\end{array}$ & $E_{50}$ & Slope & $\begin{array}{l}\mathrm{LC}_{50} / \\
\mathrm{EC}_{50} \mathrm{~d}\end{array}$ & $\begin{array}{l}\max - \\
\min (\%)\end{array}$ & BMC & $\begin{array}{l}x\left(E C_{x}\right) \text { at } \\
B M C(\%)\end{array}$ \\
\hline Azinphos-methyl & $\begin{array}{l}86-50- \\
0\end{array}$ & 2.53 & $137 \mu \mathrm{M}$ & $0.2 \mu \mathrm{M}$ & $13.6 \mu \mathrm{M}$ & $328 \mu \mathrm{M}$ & $0.7 \mu \mathrm{M}$ & 3.7 & 19 & 28 & $0.4 \mu \mathrm{M}$ & 24 \\
\hline Propoxur & $\begin{array}{l}114- \\
26-1\end{array}$ & 1.90 & $9,434 \mu \mathrm{M}$ & $42 \mu \mathrm{M}$ & $509.3 \mu \mathrm{M}$ & $1377 \mu \mathrm{M}$ & $9.8 \mu \mathrm{M}$ & 1.6 & 52 & 20 & $6.0 \mu \mathrm{M}$ & 25 \\
\hline Flucythrinate & $\begin{array}{l}70124- \\
77-5\end{array}$ & 6.56 & $89 \mathrm{nM}$ & $1 \mathrm{nM}$ & $>$ solubility & $33.5 \mathrm{nM}$ & $12.1 \mathrm{nM}$ & 1.8 & $2.8 \mathrm{~d}$ & 47 & $4.2 \mathrm{nM}$ & 25 \\
\hline Esfenvalerate & \begin{tabular}{|l}
$66230-$ \\
$04-4$
\end{tabular} & 6.76 & $15 \mathrm{nM}$ & $3.6 \mathrm{nM}$ & $>$ solubility & $21.3 \mathrm{nM}$ & $1.1 \mathrm{nM}$ & 2.4 & $19 d$ & 36 & $0.6 \mathrm{nM}$ & 26 \\
\hline Tricaine & \begin{tabular}{|l|}
$886-$ \\
$86-2$
\end{tabular} & 1.80 & $11,496 \mu \mathrm{M}$ & $302 \mu \mathrm{M}$ & $387.4 \mu \mathrm{M}$ & $1730 \mu \mathrm{M}$ & $58.3 \mu \mathrm{M}$ & 1 & 6.5 & 67 & $14.8 \mu \mathrm{M}$ & 33 \\
\hline 3,4-Dichloroaniline & \begin{tabular}{|l}
$95-$ \\
$76-1$
\end{tabular} & 2.37 & $2,086 \mu \mathrm{M}$ & $47 \mu \mathrm{M}$ & $11.8 \mu \mathrm{M}$ & $472 \mu \mathrm{M}$ & - & - & - & - & $4.5 \mu \mathrm{M}$ & - \\
\hline
\end{tabular}

${ }^{a}$ Estimated with EPI Suite ${ }^{\mathrm{TM}}$ - Estimation Program Interface v4.11, available at https://www.epa.gov/tsca-screening-tools/download-episuitetm-estimation-program-interface-v411; ${ }^{b}$ Russom et al. (1997); ${ }^{\circ}$ Calculated based on Klüver et al. (2016); ${ }^{d}$ For compounds not causing lethal effects, the ratio of $\mathrm{FET} \mathrm{LC} 50 / \mathrm{PMR} \mathrm{EC}_{50}$ was calculated using the baseline toxicity of fish embryos instead of the LC $\mathrm{L}_{50}$. 


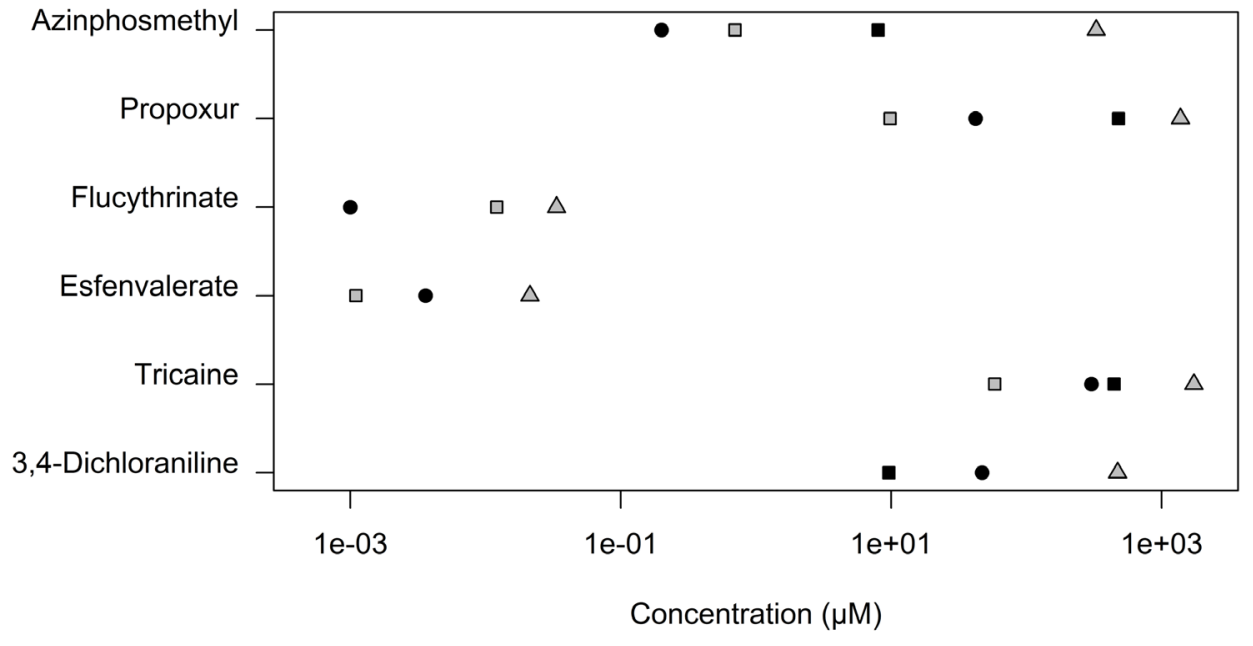

Adult fish

- LC50 - AFT
Fish embryo

- LC50 - FET

EC50 - PMR

$\triangle$ LC50 - Baseline toxicity
Fig. 4: Graph with an overview of the different effect concentrations of the test compounds Depicted are AFT $\mathrm{LC}_{50}$ values $(96 \mathrm{~h}$; fathead minnow), $\mathrm{LC}_{50}$ FET (48 h), predicted baseline toxicities for the zebrafish embryo, and $\mathrm{EC}_{50}$ values for PMR effects in zebrafish embryos. In the FET test, no mortality was observed for esfenvalerate and flucythrinate at maximum concentrations that were in the range of water solubility.
$\mathrm{LC}_{50}(48 \mathrm{~h})$ values for those compounds cannot serve to predict AFT effect concentrations. The FET $\mathrm{LC}_{50}(48 \mathrm{~h})$ value of propoxur was in the same range as baseline toxicity estimates. No mortality in FET tests was seen for flucythrinate and esfenvalerate up to concentrations close to their baseline toxicity estimates that were above their water solubilities (Tab. 1). It thus can be assumed that the lethal effects of propoxur, flucythrinate, and esfenvalerate in the FET test are due to a narcotic MoA or baseline toxicity, respectively. For azinphos-methyl, the FET $\mathrm{LC}_{50}(96 \mathrm{~h} ; 10.7 \mu \mathrm{M})$ was found to be considerably below baseline toxicity for zebrafish embryos $(274.5 \mu \mathrm{M})$ as already reported by Klüver et al. (2016). The authors considered oxidative stress and not neurotoxicity by this compound as the MoA causing mortality. In contrast, AFT, FET and baseline toxicity effect concentrations of tricaine were all in the same order of magnitude (Tab. 1). This difference between tricaine and the other test compounds can be explained by differences in the MoA and the related impact on oxygen demand. Tricaine, a voltagegated sodium channel antagonist, causes reduced muscular activity. In contrast, AChE inhibitors and voltage-gated sodium activators cause enhanced muscular activity, increasing the demand for oxygen/energy. The respiratory failure syndrome by those compounds may therefore be more severe in adult fish, resulting in comparatively low AFT lethal effect values. For 3,4-dichloroaniline, AFT and FET $\mathrm{LC}_{50}$ values were below baseline toxicity, indicating a specific rather than a narcotic MoA of this compound (Klüver et al., 2016). When embryos were exposed to 3,4-dichloroaniline, PMR effects only occurred in the lethal effect concentration range, probably reflecting unspecific responses related to overt toxicity (Tab. 1).

\subsection{Concentration dependency of the PMR patterns}

While PMR activity parameters determined with 2D-density analysis are suitable for determining PMR effect concentrations, they do not provide detailed qualitative information on PMR movement patterns in specific PMR phases. In previous studies, movement pattern analysis was used to distinguish between MoA-related movement patterns within PMR subphases based on cluster analysis (Copmans et al., 2016; Kokel et al., 2010). In those studies, however, a single or limited number of concentrations were tested, and a potential concentration dependency of PMR effects was not considered.

The concentration dependency of PMR movement found here indicates that, dependent on the PMR phase, the effect direction can change from increasing to decreasing movement activity. This was particularly observed for the AChE inhibitors propoxur and azinphos-methyl (Tab. S4 ${ }^{1}$ ). Given that the change from increased to decreased movement activity was observed at concentrations close to lethality, the decreased movement activity may reflect intense muscular seizures or unspecific effects due to overt toxicity. For esfenvalerate and flucythrinate, the concentration-dependent change from increased to decreased movement in certain subphases of the PMR was less pronounced. However, no mortality was observed for these compounds due to water solu- 


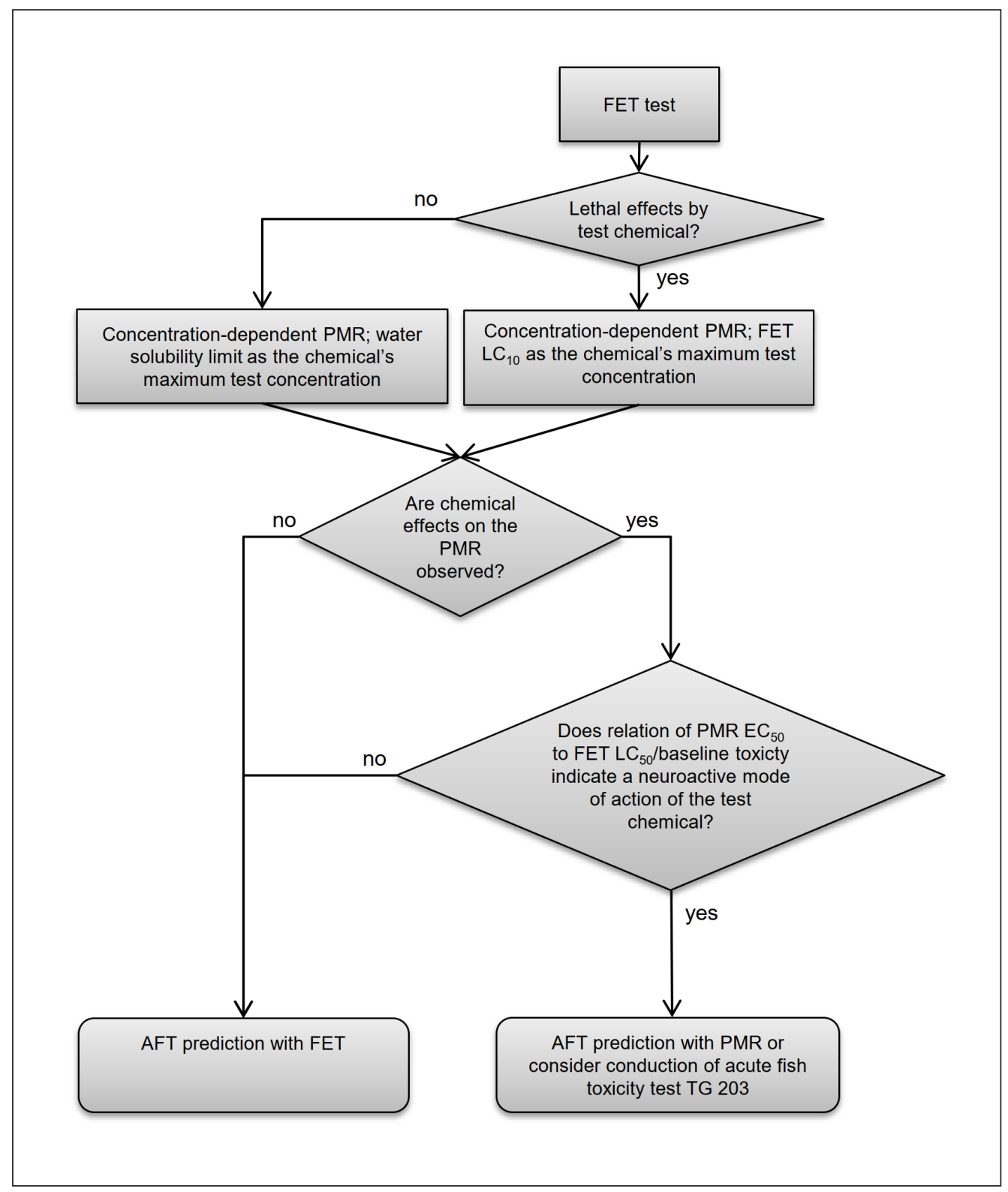

Fig. 5: Proposed workflow

for assessing the AFT of neuroactive chemicals based on FET and PMR data PMR effect concentrations could be used to predict AFT lethal concentrations of neuroactive compounds. For compounds that are not neuroactive or for which PMR fingerprints rather indicate an unspecific secondary response (refer to Fig. 3 ), the AFT is predicted based on the FET effect concentration.

bility limitations and, thus, unspecific effects may not affect the PMR responses.

It is obvious from our data that movement patterns can change with concentration. Hence, it would be necessary to include concentration-dependency in pattern analysis and relate the effects to toxicity for a diagnostic assessment.

\section{Outlook - Complementing the FET assay with PMR analysis}

Discrepancies between AFT and FET effect concentrations were found to be particularly large for neuroactive compounds (Knöbel et al., 2012; Klüver et al., 2015; Sobanska et al., 2018; Gla- berman et al., 2017). For predicting the AFT effect concentrations of neuroactive compounds with the zebrafish embryo, we propose to complement the FET assay with a quantitative measurement of the behavior, such as the presented approach for determining PMR effect concentrations. PMR effects could be used to increase the predictive capacity of the FET for AFT by inferring AFT levels from PMR effect concentrations of neurotoxic compounds (refer to Fig. 5). For the use of the PMR for diagnostic assessment, it is also important to consider concentration-dependency of the PMR response and confounding effects on the behavior related to overt toxicity.

To confirm that the proposed combination of FET and PMR assays provides robust data that reliably corresponds with AFT effect concentrations of neuroactive compounds, further research 
should address 1) whether PMR responses determined across different labs are comparable and 2) whether stage-dependent differences in absorption, distribution, metabolism, and elimination (ADME) of chemicals could have an impact on effect concentrations and confound the detection of neuroactive compounds.

\section{References}

Attili, S. and Hughes, S. M. (2014). Anaesthetic tricaine acts preferentially on neural voltage-gated sodium channels and fails to block directly evoked muscle contraction. PLoS One 9, e103751. doi:10.1371/journal.pone.0103751

Basnet, R. M., Zizioli, D., Taweedet, S. et al. (2019). Zebrafish larvae as a behavioral model in neuropharmacology. Biomedicines 7, 23. doi:10.3390/biomedicines7010023

Belanger, S. E., Rawlings, J. M. and Carr, G. J. (2013). Use of fish embryo toxicity tests for the prediction of acute fish toxicity to chemicals. Environ Toxicol Chem 32, 1768-1783. doi:10.1002/etc. 2244

Bradbury, S. P., Carlson, R. W., Henry, T. R. et al. (2008). Toxic responses of the fish nervous system. In R. T. Di Giulio and D. E. Hinton (eds.), The Toxicology of Fishes. CRC Press. doi:10.1201/9780203647295

Brox, S., Seiwert, B., Haase, N. et al. (2016). Metabolism of clofibric acid in zebrafish embryos (Danio rerio) as determined by liquid chromatography-high resolution-mass spectrometry. Comp Biochem Physiol C Toxicol Pharmacol 185-186, 20-28. doi:10.1016/j.cbpc.2016.02.007

Buckingham, S. D. and Ali, D. W. (2004). Sodium and potassium currents of larval zebrafish muscle fibres. J Exp Biol 207, 841852. doi:10.1242/jeb.00839

Busquet, F., Strecker, R., Rawlings, J. M. et al. (2014). OECD validation study to assess intra- and inter-laboratory reproducibility of the zebrafish embryo toxicity test for acute aquatic toxicity testing. Regul Toxicol Pharmacol 69, 496-511. doi:10.1016/j.yrtph.2014.05.018

Copmans, D., Meinl, T., Dietz, C. et al. (2016). A KNIME-based analysis of the zebrafish photomotor response clusters the phenotypes of 14 classes of neuroactive molecules. J Biomol Screen 21, 427-436. doi:10.1177/1087057115618348

Coutts, C. A., Patten, S. A., Balt, L. N. et al. (2006). Development of ionic currents of zebrafish slow and fast skeletal muscle fibers. J Neurobiol 66, 220-235. doi:10.1002/neu.20214

D'Amora, M. and Giordani, S. (2018). The utility of zebrafish as a model for screening developmental neurotoxicity. Front Neurosci 12, 976. doi:10.3389/fnins.2018.00976

de Brujin, M. H., Labrada, L. A., Smyth, A. J. et al. (1993). A comparative study of diagnosis by the polymerase chain reaction and by current clinical methods using biopsies from colombian patients with suspected leishmaniasis. Trop Med Parasitol 44, 201-207.

Ducharme, N. A., Peterson, L. E., Benfenati, E. et al. (2013). Meta-analysis of toxicity and teratogenicity of 133 chemicals from zebrafish developmental toxicity studies. Reprod Toxicol 41, 98-108. doi:10.1016/j.reprotox.2013.06.070

Embry, M. R., Belanger, S. E., Braunbeck, T. A. et al. (2010).
The fish embryo toxicity test as an animal alternative method in hazard and risk assessment and scientific research. Aquat Toxicol 97, 79-87. doi:10.1016/j.aquatox.2009.12.008

Fukuto, T. R. (1990). Mechanism of action of organophosphorus and carbamate insecticides. Environ Health Perspect 87, 245 254. doi:10.1289/ehp.9087245

Gauthier, P. T. and Vijayan, M. M. (2018). Nonlinear mixed-modelling discriminates the effect of chemicals and their mixtures on zebrafish behavior. Sci Rep 8, 1999. doi:10.1038/s41598018-20112-X

Glaberman, S., Padilla, S. and Barron, M. G. (2017). Evaluating the zebrafish embryo toxicity test for pesticide hazard screening. Environ Toxicol Chem 36, 1221-1226. doi:10.1002/etc.3641

Gruber, S. J. and Munn, M. D. (1998). Organophosphate and carbamate insecticides in agricultural waters and cholinesterase (ChE) inhibition in common carp (Cyprinus carpio). Arch Environ Contam Toxicol 35, 391-396. doi:10.1007/ s002449900393

Hanneman, E. and Westerfield, M. (1989). Early expression of acetylcholinesterase activity in functionally distinct neurons of the zebrafish. J Comp Neurol 284, 350-361. doi:10.1002/ cne.902840303

Herzsprung, P., Weil, L. and Niessner, R. (1992). Measurement of bimolecular rate constants $\mathrm{k}(\mathrm{i})$ of the cholinesterase inactivation reaction by 55 insecticides and of the influence of various pyridiniumoximes on k(i). Int J Environ Anal Chem 47, 181-200. doi:10.1080/03067319208027028

Jacob, E., Drexel, M., Schwerte, T. et al. (2002). Influence of hypoxia and of hypoxemia on the development of cardiac activity in zebrafish larvae. Am J Physiol Regul Integr Comp Physiol 283, R911-917. doi:10.1152/ajpregu.00673.2001

Klüver, N., König, M., Ortmann, J. et al. (2015). Fish embryo toxicity test: Identification of compounds with weak toxicity and analysis of behavioral effects to improve prediction of acute toxicity for neurotoxic compounds. Environ Sci Technol 49, 7002-7011. doi:10.1021/acs.est.5b01910

Klüver, N., Vogs, C., Altenburger, R. et al. (2016). Development of a general baseline toxicity QSAR model for the fish embryo acute toxicity test. Chemosphere 164, 164-173. doi:10.1016/j. chemosphere.2016.08.079

Knöbel, M., Busser, F. J., Rico-Rico, A. et al. (2012). Predicting adult fish acute lethality with the zebrafish embryo: Relevance of test duration, endpoints, compound properties, and exposure concentration analysis. Environ Sci Technol 46, 96909700. doi:10.1021/es301729q

Kokel, D., Bryan, J., Laggner, C. et al. (2010). Rapid behaviorbased identification of neuroactive small molecules in the zebrafish. Nat Chem Biol 6, 231-237. doi:10.1038/nchembio. 307

Kokel, D., Cheung, C. Y., Mills, R. et al. (2013a). Photochemical activation of trpal channels in neurons and animals. Nat Chem Biol 9, 257-263. doi:10.1038/nchembio. 1183

Kokel, D., Dunn, T. W., Ahrens, M. B. et al. (2013b). Identification of nonvisual photomotor response cells in the vertebrate hindbrain. J Neurosci 33, 3834-3843. doi:10.1523/jneurosci. 3689-12.2013 
Lammer, E., Carr, G. J., Wendler, K. et al. (2009a). Is the fish embryo toxicity test (FET) with the zebrafish (Danio rerio) a potential alternative for the fish acute toxicity test? Comp Biochem Physiol C Toxicol Pharmacol 149, 196-209. doi:10.1016/j.cbpc.2008.11.006

Lammer, E., Kamp, H.G., Hisgen, V. et al. (2009b). Development of a flow-through system for the fish embryo toxicity test (FET) with the zebrafish (Danio rerio). Toxicol In Vitro 23, 1436-1442. doi:10.1016/j.tiv.2009.05.014

Legradi, J., el Abdellaoui, N., van Pomeren, M. et al. (2015). Comparability of behavioural assays using zebrafish larvae to assess neurotoxicity. Environ Sci Pollut Res Int 22, $16277-$ 16289. doi:10.1007/s11356-014-3805-8

Nagel, R. (2002). Dart: The embryo test with the zebrafish Danio rerio - A general model in ecotoxicology and toxicology. ALTEX 19, Suppl 1, 38-48. https://www.altex.org/index.php/ altex/article/view/2125

OECD (2013). Test No. 236: Fish Embryo Acute Toxicity (FET) Test. OECD Guidelines for the Testing of Chemicals, Section 2. OECD Publishing, Paris. doi:10.1787/9789264203709-en

OECD (2019). Test No. 203: Fish, Acute Toxicity Test. OECD Guidelines for the Testing of Chemicals, Section 2. OECD Publishing, Paris. doi:10.1787/9789264069961-en

Ogungbemi, A., Leuthold, D., Scholz, S. et al. (2019). Hypo- or hyperactivity of zebrafish embryos provoked by neuroactive substances: A review on how experimental parameters impact the predictability of behavior changes. Environ Sci Eur 31, 88. doi:10.1186/s12302-019-0270-5

Reif, D. M., Truong, L., Mandrell, D. et al. (2016). High-throughput characterization of chemical-associated embryonic behavioral changes predicts teratogenic outcomes. Arch Toxicol 90, 1459-1470. doi:10.1007/s00204-015-1554-1

Rombough, P. (2002). Gills are needed for ionoregulation before they are needed for $\mathrm{o}_{2}$ uptake in developing zebrafish, Danio rerio. J Exp Biol 205, 1787-1794. doi:10.1242/jeb.205.12.1787

Russom, C. L., Bradbury, S. P., Broderius, S. J. et al. (1997). Predicting modes of toxic action from chemical structure: Acute toxicity in the fathead minnow (Pimephales promelas). Environ Toxicol Chem 16, 948-967. doi:10.1002/etc.5620160514

Russom, C. L., LaLone, C. A., Villeneuve, D. L. et al. (2014). Development of an adverse outcome pathway for acetylcholinesterase inhibition leading to acute mortality. Environ Toxicol Chem 33, 2157-2169. doi:10.1002/etc. 2662

Saeed, A. I., Sharov, V., White, J. et al. (2003). Tm4: A free, open-source system for microarray data management and analysis. Biotechniques 34, 374-378. doi:10.2144/03342mt01

Scholz, S., Fischer, S., Gündel, U. et al. (2008). The zebrafish embryo model in environmental risk assessment - Applications beyond acute toxicity testing. Environ Sci Pollut Res Int 15, 394-404. doi:10.1007/s11356-008-0018-z

Scholz, S., Sela, E., Blaha, L. et al. (2013). A European perspective on alternatives to animal testing for environmental hazard identification and risk assessment. Regul Toxicol Pharmacol 67, 506-530. doi:10.1016/j.yrtph.2013.10.003

Selderslaghs, I. W., Hooyberghs, J., De Coen, W. et al. (2010). Locomotor activity in zebrafish embryos: A new method to assess developmental neurotoxicity. Neurotoxicol Teratol 32, 460-471. doi:10.1016/j.ntt.2010.03.002

Selderslaghs, I. W., Hooyberghs, J., Blust, R. et al. (2013). Assessment of the developmental neurotoxicity of compounds by measuring locomotor activity in zebrafish embryos and larvae. Neurotoxicol Teratol 37, 44-56. doi:10.1016/j.ntt.2013.01.003

Sobanska, M., Scholz, S., Nyman, A. M. et al. (2018). Applicability of the fish embryo acute toxicity (FET) test (OECD 236) in the regulatory context of registration, evaluation, authorisation, and restriction of chemicals (REACH). Environ Toxicol Chem 37, 657-670. doi:10.1002/etc.4055

Soderlund, D. M. and Bloomquist, J. R. (1989). Neurotoxic actions of pyrethroid insecticides. Annu Rev Entomol 34, 77-96. doi:10.1146/annurev.en.34.010189.000453

Strähle, U., Scholz, S., Geisler, R. et al. (2012). Zebrafish embryos as an alternative to animal experiments - A commentary on the definition of the onset of protected life stages in animal welfare regulations. Reprod Toxicol 33, 128-132. doi:10.1016/j.reprotox.2011.06.121

Teixido, E., Kießling, T. R., Krupp, E. et al. (2019). Automated morphological feature assessment for zebrafish embryo developmental toxicity screens. Toxicol Sci 167, 438-449. doi:10.1093/toxsci/kfy250

Venables, W. N. and Ripley, B. D. (2002). Modern Applied Statistics with S. Statistics and Computing. New York, NY, USA: Springer. doi:10.1007/978-0-387-21706-2

\section{Conflict of interest}

We declare that none of the authors has a conflict of interest.

\section{Acknowledgements}

We would like to thank Silke Aulhorn, Janet Krüger and David Leuthold for their excellent technical support regarding FET assays. This work was part of the research topic "Chemicals in the Environment" (CITE) within the research program of the UFZ - Helmholtz Centre for Environmental Research (POFIIIExposome). 